\title{
The Persistence of Invasion and Diffusion Model of Poisonous Weeds with Allee Effect
}

\author{
Lei Shi ${ }^{1}$, Hua Liu ${ }^{1, *}$, Yumei Wei ${ }^{2}$, Ming $\mathrm{Ma}^{1}$ and Rui Jiang ${ }^{3}$ \\ ${ }^{1}$ School of Mathematics and Computer Science, Northwest Minzu University, Lanzhou, 730030, Gansu, China \\ ${ }^{2}$ Experimental Center, Northwest Minzu University, Lanzhou, 730030, Gansu, China \\ ${ }^{3}$ Chongqing Medical and Health School, Fuling, 408100, Chongqing, China \\ ${ }^{*}$ Corresponding author
}

\begin{abstract}
In this paper, a water resource competition model of poisonous weed invasion and diffusion with Allee effect was studied. We first discuss the persistence of the model. After that, by discretizing the model, the population dynamics with and without Allee effect was simulated. The results shows that: 1 . The Allee effect make it more difficult to reach persistence coexistence of edible grass and poisonous weeds; 2 . The Allee effect leads to the dynamic change of population quantity from periodic coexistence to stability of poisonous weeds reaches saturation and edible grass becomes extinction.
\end{abstract}

Keywords-competition; invasion; permanence

\section{INTRODUCTION}

Traditional interspecific competitive model of the root system competes with soil water takes the form as follows [1,2]

$$
\left\{\begin{array}{l}
\frac{d x_{i}}{d t}=r_{1} x_{i}\left(1-\frac{x_{i}}{K_{1}}-\alpha \frac{\lambda_{2}}{r_{1}} \frac{V}{V_{1}} y_{i}\right) \\
\frac{d y_{i}}{d t}=r_{2} y_{i}\left(1-\frac{y_{i}}{K_{2}}-\beta \frac{\lambda_{1}}{r_{2}} \frac{V}{V_{2}} x_{i}\right)
\end{array}\right.
$$

Where $r_{1}>0, r_{2}>0, \alpha>0, \beta>0,0<\lambda_{1}<1,0<\lambda_{2}<1$, and $K_{i}, i=1,2$ is the carrying capacity[3]. $V_{i}(i=1,2)$ is the water resource warehouse of edible grass and poisonous weeds, $V=V_{1} \cap V_{2}$ is the overlapping parts of the two water resource warehouse( they mutual competition resource in $V$ ). The parameter $\lambda_{1}, \lambda_{2}$ is respectively express utilization of water resources for edible forage and poisonous weeds, $\lambda_{1}+\lambda_{2}=1$. The parameter $\alpha, \beta$ respectively indicates the coefficient of proportionality of moisture loss in root soil layer caused by edible forage and poisonous weeds consumes water resources.

And the local asymptotic stability of the model is given in [4], but this model does not consider Allee effect and intrusion diffusion.

In this article, the most representative expression of Allee effect is $\left(1-\frac{A+c}{x_{i}+c}\right)$, among $A$ is the Allee threshold ( $A>0$ is the strong Allee effect, $A<0$ is the feeble Allee effect), $C$ is the auxiliary parameter $(c>0, c \geq-A)$. When $A+c=0$, the Allee effect expression value is 1 and that indicate the forage grass had no Allee effect. The parameter $C$ affects the growth curve of edible grass, the curve will flatten when it increases. When $x_{i}<A$, the value of Allee effect is less than zero, it shows that the population density of edible forage is less than A of negative population growth; When $x_{i}>A$, the value of Allee effect is greater than zero, it shows that the population density of edible forage is greater than $A$ of positive population growth [5].

For the intrusion diffusion, many scholars have done a lot of research, and given many forms of diffusion. In [6], Takeuchi Y took into account the two patch system connected by diffusion, and the diffusion term given was $\varepsilon\left(x_{j}-x_{i}\right)$, $\delta\left(y_{j}-y_{i}\right)$. In [7], Zhang Xinan proposed the competitive Lotka-Volterra model that only one species can diffuse between the two patches, and the diffusion term given was $\varepsilon\left(x_{2}-x_{1}\right)$. This article will give the diffusion term as $\mu\left(y_{j}-y_{i}\right)$. The diffusion term reflects the invasion and diffusion of $y_{j}$ patches of poisonous weeds' neighbor to the $y_{i}$ patches and $\mu$ is the diffusion coefficient [6, 7].

On this basis, a water resources competition model with Allee effect and the diffusion term is established, as follows

$$
\left\{\begin{array}{l}
\frac{d x_{i}}{d t}=r_{1} x_{i}\left(\left(1-\frac{x_{i}}{K_{1}}\right)\left(1-\frac{A+c}{x_{i}+c}\right)-\alpha \frac{\lambda_{2} V}{r_{1} V_{1}} y_{i}\right) \\
\frac{d y_{i}}{d t}=r_{2} y_{i}\left(1-\frac{y_{i}}{K_{2}}-\beta \frac{\lambda_{1}}{r_{2}} \frac{V}{V_{2}} x_{i}\right)+\mu\left(y_{j}-y_{i}\right)
\end{array}\right.
$$

\section{MAIN RESUlT}

Persistence is an important basis for the long-term coexistence of edible forage and poisonous weeds in arid and semi-arid environments. The extinction of edible grass due to various causes is bound to make poisonous weeds run riot owing to loss of competitors. This will destroy the edible forage and poisonous weeds competition and coexistence, thereby breaking the ecological diversity of natural grassland, further to the development of animal husbandry in the 
devastating disaster. Moreover, the extinction of poisonous weed is bound to affect the growth of edible grass.

Now let us state several concepts and lemmas which will be useful in the proving of main results.

\section{A. The Basic Concept}

Definition1: For a continuous time system $x(t)=f(t, x)$, if there are constant $m, M_{1}>0$ let each positive solution $X\left(t, t_{0}, \varphi\right)=\left(x_{1}(t), x_{2}(t), \cdots, x_{n}(t)\right)$ of the system is satisfied

$$
0<m \leq \lim _{t \rightarrow+\infty} \inf x_{i}(t) \leq \lim _{t \rightarrow+\infty} \sup x_{i}(t) \leq M, i=1,2, \cdots, n,
$$

then system is persistent, otherwise known as Non persistent.

Lemma 1 (Comparison principle) [8]: Assuming that $f(t, x)$ and $F(t, x)$ are continuous on the planar region $G$, also satisfied $f(t, x) \leq F(t, x)$, if $(t, \xi) \in G$ and $\phi(t), \varphi(t)$ is respectively the unique solution of $\left\{\begin{array}{l}x(t)=f(t, x) \\ x(\tau)=\xi\end{array}\right.$ and $\left\{\begin{array}{l}\dot{x}(t)=F(t, x) \\ x(\tau)=\xi\end{array}\right.$ defined on $a<t<b$, then

1) if $\tau \leq t<b$, about $\varphi(t) \leq \phi(t)$;

2) if $a \leq t<\tau$, about $\varphi(t) \geq \phi(t)$.

Lemma 2[9]: If $a>0, b>0$ and $\frac{d x(t)}{d t} \geq x(t)(b-a x(t))$, when $t \geq 0, x(0)>0$, we have $\lim _{t \rightarrow+\infty} \inf x(t) \geq \frac{b}{a} \quad$;f $a>0, b>0$ and $\frac{d x(t)}{d t} \leq x(t)(b-a x(t))$, when $t \geq 0$, $x(0)>0$, we have $\lim _{t \rightarrow+\infty} \sup x(t) \leq \frac{b}{a}$.

The scope of the model (2) discussion is $R_{+}^{2}=\left\{x_{i} \geq 0, y_{i} \geq 0\right\}$ based on practical significance.

Theorem 1: If

$$
\begin{gathered}
\frac{K_{1}\left(K_{1}+c\right)}{A-K_{1}}\left(1-\frac{A+c}{K_{1}+c}-\delta_{1}\left(\frac{K_{2}}{\mu K_{2}+1}+I\right)\right)>0, \\
\frac{K_{2}}{\mu K_{2}+r_{2}}\left(r_{2}-r_{2} \delta_{2} K_{1}\right)+I>0,
\end{gathered}
$$

then model (2) is persistent.

Proof: From the first equation of model (2) it follows that

$$
\begin{aligned}
& \frac{d x_{i}}{d t}=r_{1} x_{i}\left(\left(1-\frac{x_{i}}{K_{1}}\right)\left(1-\frac{A+c}{x_{i}+c}\right)-\alpha \frac{\lambda_{2} V}{r_{1} V_{1}} y_{i}\right) \\
\leq & x_{i}\left(r_{1}\left(1-\frac{x_{i}}{K_{1}}\right)-r_{1} \alpha \frac{\lambda_{2} V}{r_{1} V_{1}} y_{i}\right) \leq x_{i}\left(r_{1}-\frac{r_{1}}{K_{1}} x_{i}\right),
\end{aligned}
$$

Again, according to the comparison principle of differential equation and Lemma 2, one has

$$
\lim _{t \rightarrow+\infty} \sup x_{i}(t) \leq K_{1}=\bar{x},
$$

For any positive constant $\varepsilon>0$, exists a $T_{1}>0$ such that for $t>T_{1}$,

$$
x_{i}(t) \leq \bar{x}+\varepsilon
$$

Same above, According to the first equation of model (2) leads to

$$
\begin{gathered}
\frac{d y_{i}}{d t}=r_{2} y_{i}\left(\frac{K_{2}-y_{i}-\beta \lambda_{1} \frac{K_{2}}{r_{2}} \frac{V}{V_{2}} x_{i}}{K_{2}}\right)+\mu\left(y_{j}-y_{i}\right) \\
=r_{2} y_{i}\left(1-\left(\frac{1}{K_{2}}+\mu\right) y_{i}-\beta \frac{\lambda_{1}}{r_{2}} \frac{V}{V_{2}} x_{i}\right)+I \\
\leq y_{i}\left(r_{2}-\left(\frac{r_{2}}{K_{2}}+r_{2} \mu\right) y_{i}\right)+I,
\end{gathered}
$$

Again, by applying Lemma 2, one obtains

$$
\limsup _{t \rightarrow+\infty} y_{i}(t) \leq \frac{r_{2}}{\frac{r_{2}}{K_{2}}+r_{2} \mu}+I=\frac{K_{2}}{\mu K_{2}+1}+I=\bar{y}
$$

So, for any positive constant $\varepsilon>0$, exists $T_{1}>0$ such that for $t>T_{1}$,

$$
x_{i}(t) \leq \bar{x}+\varepsilon, y_{i}(t) \leq \bar{y}+\varepsilon
$$

On the other hand, one obtains:

$$
\begin{gathered}
\frac{d x_{i}}{d t} \geq x_{i}\left(r_{1}\left(1-\frac{x_{i}}{K_{1}}\right)\left(1-\frac{A+c}{\bar{x}+c}\right)-r_{1} \alpha \frac{\lambda_{2} V}{r_{1} V_{1}}(\bar{y}+\varepsilon)\right), \\
\frac{d y_{i}}{d t} \geq y_{i}\left(r_{2}-\left(\mu+\frac{r_{2}}{K_{2}}\right) y_{i}-r_{2} \beta \frac{\lambda_{1} V}{r_{2} V_{2}}(\bar{x}+\varepsilon)\right)+I,
\end{gathered}
$$


By applying Lemma 1 and Lemma 2, one has

$$
\begin{gathered}
\lim _{t \rightarrow+\infty} \inf x_{i}(t) \geq \frac{1}{\frac{A+c}{K_{1}(\bar{x}+c)}-\frac{1}{K_{1}}} \\
\cdot\left(r_{1}-r_{1} \frac{A+c}{\bar{x}+c}-r_{1} \alpha \frac{\lambda_{2} V}{r_{1} V_{1}}(\bar{y}+\varepsilon)\right) \\
=\frac{K_{1}(\bar{x}+c)}{A-\bar{x}}\left(r_{1}-r_{1} \frac{A+c}{\bar{x}+c}-r_{1} \delta_{1}(\bar{y}+\varepsilon)\right) \\
\liminf _{t \rightarrow+\infty} y_{i}(t) \geq \frac{K_{2}}{\mu K_{2}+r_{2}}\left(r_{2}-r_{2} \beta \frac{\lambda_{1} V}{r_{2} V_{2}}(\bar{x}+\varepsilon)\right)+I \\
=\frac{K_{2}}{\mu K_{2}+r_{2}}\left(r_{2}-r_{2} \delta_{2}(\bar{x}+\varepsilon)\right)+I,
\end{gathered}
$$

Here $\delta_{1}=\alpha \frac{\lambda_{2}}{r_{1}} \frac{V}{V_{1}}, \delta_{2}=\beta \frac{\lambda_{1}}{r_{2}} \frac{V}{V_{2}}$,

Setting $\varepsilon \rightarrow 0$ in above inequalities leads to

$$
\begin{gathered}
\lim _{t \rightarrow+\infty} \inf x_{i}(t) \geq \frac{K_{1}(\bar{x}+c)}{A-\bar{x}}\left(r_{1}-r_{1} \frac{A+c}{\bar{x}+c}-r_{1} \delta_{1} \bar{y}\right) \\
=\frac{r_{1} K_{1}\left(K_{1}+c\right)}{A-K_{1}}\left(1-\frac{A+c}{K_{1}+c}-\delta_{1}\left(\frac{K_{2}}{\mu K_{2}+1}+I\right)\right) \\
=\underline{x}, \\
\lim _{t \rightarrow+\infty} \inf y_{i}(t) \geq \frac{K_{2}}{\mu K_{2}+r_{2}}\left(r_{2}-r_{2} \delta_{2} \underline{x}\right)+I \\
=\frac{K_{2}}{\mu K_{2}+r_{2}}\left(r_{2}-r_{2} \delta_{2} K_{1}\right)+I=\underline{y},
\end{gathered}
$$

From Definition 1,

$$
\begin{gathered}
0<m \leq \underline{x}=\frac{r_{1} K_{1}\left(K_{1}+c\right)}{A-K_{1}}\left(1-\frac{A+c}{K_{1}+c}-\delta_{1}\left(\frac{K_{2}}{\mu K_{2}+1}+I\right)\right) \\
\leq \bar{x}=K_{1} \leq M,
\end{gathered}
$$

$0<m \leq \underline{y}=\frac{K_{2}}{\mu K_{2}+r_{2}}\left(r_{2}-r_{2} \delta_{2} K_{1}\right)+I \leq \bar{y}=\frac{K_{2}}{\mu K_{2}+1}+I \leq M$

Above inequality shows that model (2) is persistent when

$$
\begin{gathered}
\frac{r_{1} K_{1}\left(K_{1}+c\right)}{A-K_{1}}\left(1-\frac{A+c}{K_{1}+c}-\delta_{1}\left(\frac{K_{2}}{\mu K_{2}+1}+I\right)\right)>0, \\
\frac{K_{2}}{\mu K_{2}+r_{2}}\left(r_{2}-r_{2} \delta_{2} K_{1}\right)+I>0 .
\end{gathered}
$$

The proof of the theorem is complete.

\section{NUMERICAL EXAMPLE}

Discretize the model, the population dynamics with and without Allee effect was simulated with time t as the horizontal axis, the population of edible herbage is the vertical axis. Let $r_{1}=0.4, r_{2}=0.8, \mu=0.25, A=0.35, c=0.15, K_{1}=1.2$, $K_{2}=1.5, \alpha=0.4, \beta=0.8, \lambda_{1}=\sin ^{2} t, \lambda_{2}=\cos ^{2} t$, $x(0)=0.6, y(0)=0.7, \frac{V}{V_{1}}=\frac{V}{V_{2}}=0.2$

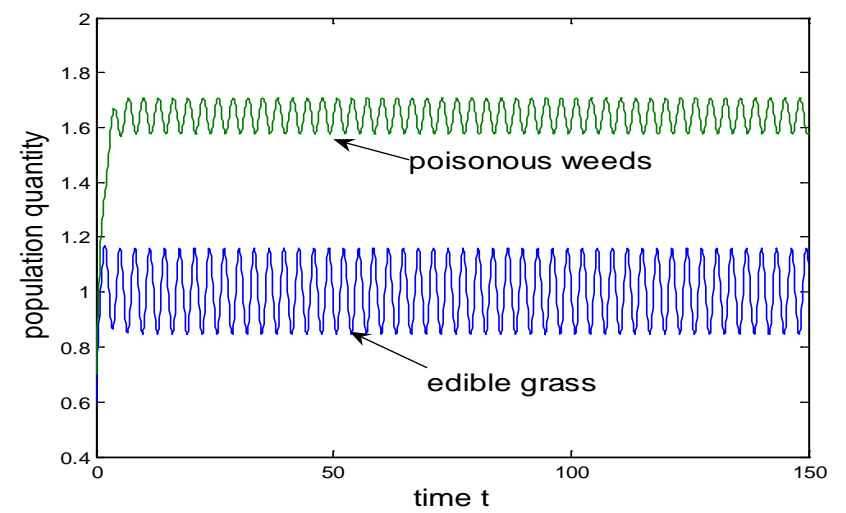

FIGURE I. THE NUMBER OF POPULATION CHANGES WITH TIME WITHOUT ALLEE EFFECT

The figure I shows: edible grasses and poisonous weeds species populations are increasing sharply in the beginning; when time $t$ is 2.5, edible grasses and poisonous weeds species populations fluctuate to gradually achieve coexistence state change trend, but the population presents the characteristics of the cycle.

Figure II shows that the edible grass populations became smaller in the beginning, and poisonous weeds population increased gradually; But when the time is 20, edible grass species populations to extinction, poisonous weeds population has reached the carrying capacity.

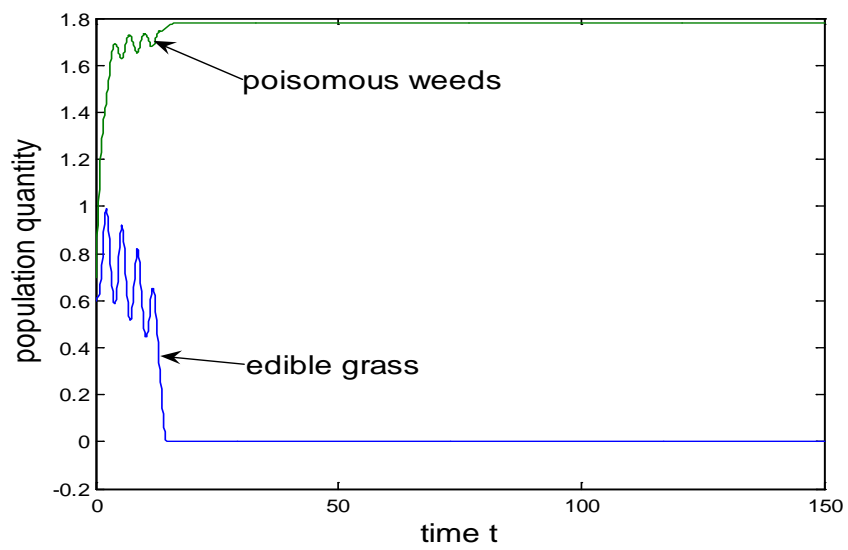

FIGURE II. THE NUMBER OF POPULATION CHANGES WITH TIME WITH ALLEE EFFECT

We can see from the above figure I and II compared to known: with the Allee effect, the original non-zero balance into edible grass the zero point of extinction, and fluctuation 
disappeared. Explain the Allee effect makes the edible grass species and poisonous weeds risk of extinction, and not conducive to edible grass species and poisonous weeds population to achieve lasting adaptation.

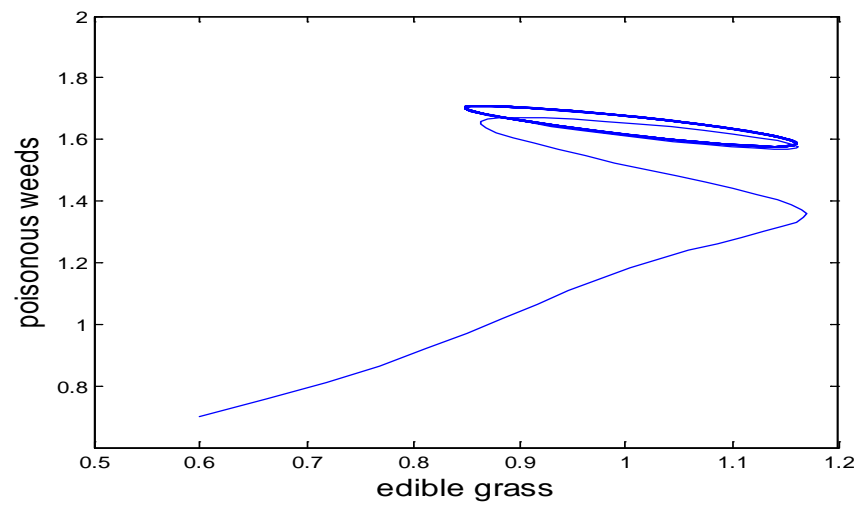

FIGURE III. WITH EDIBLE GRASS POPULATIONS FOR THE HORIZONTAL AXIS, WITH POISONOUS WEEDS POPULATIONS FOR THE LONGITUDINAL AXIS, THE SIMULATION OF THE PHASE DIAGRAM RAIL LINE WITHOUT ALLEE EFFECT

Figure III shows the changes in the phase trajectory of edible grass population and poisonous weed population without Allee effect. From figure III, it can be seen that the number of population of edible herbage population and poisonous weed population increased at the same time. When the population of edible herbage population was 1.15 and the number of poisonous weeds was 1.4, the population of edible herbage began to decrease gradually, while the number of poisonous weeds continued to increase. When the population of edible herbage was 1 and the population of the poisonous weeds was 1.65 , the two populations gradually showed a small change in cycle.

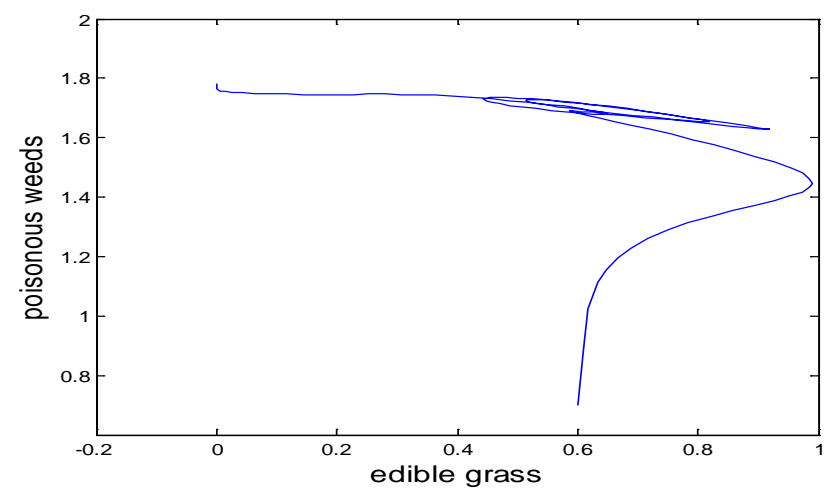

FIGURE IV. WITH EDIBLE GRASS POPULATIONS FOR THE HORIZONTAL AXIS, WITH POISONOUS WEEDS POPULATIONS FOR THE LONGITUDINAL AXIS, THE SIMULATION OF THE PHASE DIAGRAM RAIL LINE WITH ALLEE EFFECT

From figure 4, it can be seen that the number of population of edible herbage population and poisonous weed population increased at the same time. When the population of edible herbage population was 0.9 and the population of poisonous weeds was 1.4 , the population of edible herbage began to decrease gradually, while the number of poisonous weeds continued to increase. When the population of edible herbage was 0.6 , the population of the poisonous weeds was 1.7 , and the two populations gradually showed a small change in cycle. Then the population of edible herbage continued to decrease, the population of poisonous weeds continued to increase, and finally the population of herbage was extinct, and the population of poisonous weeds reached their own carrying capacity.

By contrast figure III and IV shows: the edible grasses and poisonous weeds populations appear minor cycles ahead of time with Allee effect, and edible herbage species due to the impact of Allee effect eventually tend to be extinct. Allee effect leads to population dynamic changes from periodic coexistence into poisonous weeds saturated and edible grass the stable state of extinction.

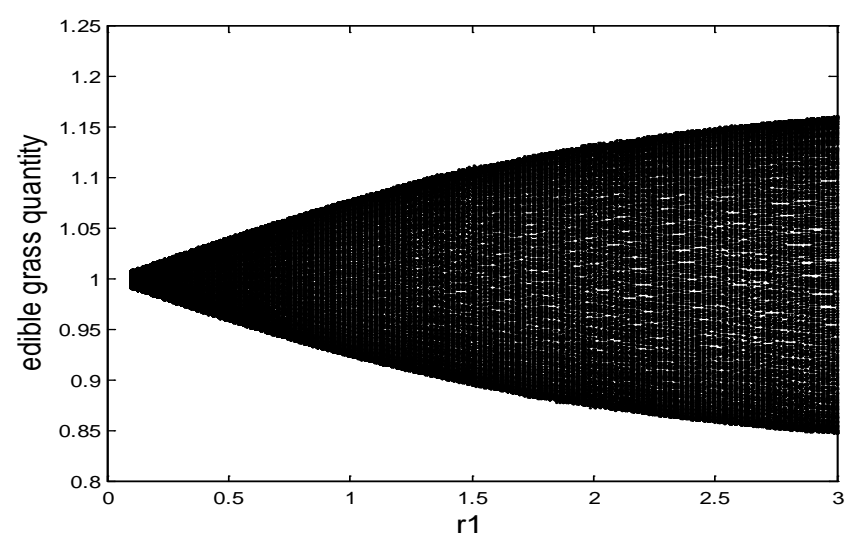

FIGURE V. WITH EDIBLE GRASSES OF THE INTRINSIC GROWTH RATE FOR THE HORIZONTAL AXIS, WITH EDIBLE FORAGE POPULATION DENSITY AS A NUMBER LINE, THE BIFURCATION DIAGRAM WITHOUT ALLEE EFFECT

Figure $\mathrm{V}$ shows that the edible grass species present range increases gradually cycle bifurcation without the Allee effect.

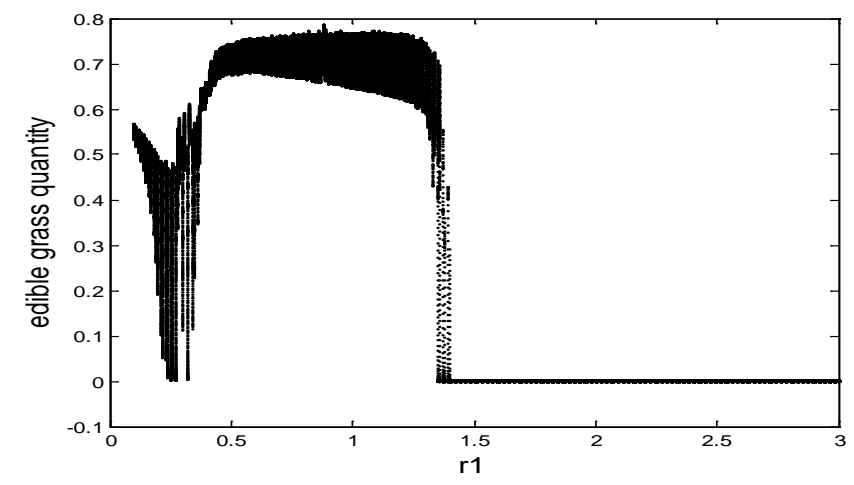

FIGURE VI. WITH EDIBLE GRASSES OF THE INTRINSIC GROWTH RATE FOR THE HORIZONTAL AXIS, WITH EDIBLE FORAGE POPULATION DENSITY AS A NUMBER LINE, THE BIFURCATION DIAGRAM WITH ALLEE EFFECT

As result, we get the conclusion by figure VI: the edible when grass intrinsic rate of population of the cycle is presented before 1.4 dynamic, after edible herbage species becomes to extinction with Allee effect. 


\section{CONCLUSION}

The Allee effect of populations have been the focus of ecology and protection of ecology, it largely increase local extinction probability, and reduce the population continue to coexist. This article discussed the edible grass with Allee effect poisonous weeds invasion diffusion competition model of water resources of locally asymptotic stability, persistence, and depict population dynamics variation of the model simulation and phase track diagram and bifurcation diagram. The study found that the Allee effect makes the co-existence of the cycle dynamic into poisonous weeds saturated and edible grass the stable state of extinction, model local asymptotic stability is more difficult to achieve. Also edible grass populations and the boundedness condition poisonous weeds population to achieve lasting adaptation are smaller, edible grasses increased risk of extinction and unfavourable edible herbage species with poisonous weeds population to achieve lasting practiced. In this paper, the numerical simulation of the final analysis also is good enough to verify the theoretical derivation of the paper.

\section{ACKNOWLEDGMENT}

This work is supported by the National Natural Science Foundation of China (31260098, 11361049, 31560127), the Program for Yong Talent of State Ethnic Affairs Commission of China (No.[2014]121), the Fundamental Research Funds for the Central Universities (31920170072, 31920170036). This work is partially supported by the first-class discipline program of Northwest Minzu University.

\section{REFERENCES}

[1] Xu Cailin. The researches on the computer modeling experiment of plant intraspecific and interspecific competition[D]. Lanzhou University. 2000.

[2] Liu H, Jin X, Shi L,et al.Spatial distribution of poisonous weed invasion based on inter-species competition models. Acta Ecologica Sinica, 2017, 37(11):3765-3775.

[3] Xu R, Chaplain MAJ, Davidson FA. Persistence and global stability of a ratio-dependent predator-prey model with stage structure. Applied Mathematics \& Computation. 2004; 158: 729-744.

[4] Shi L, Liu H, et al. The stability analysis of poisonous weeds' invasion based on the competition of water resources model. Journal of Hubei University for Nationalities(Natural Science Edition), 2016,34(4):365370.

[5] Sun GQ. Mathematical modeling of population dynamics with Allee effect. Nonlinear Dynamics. 2016: 1-12.

[6] Takeuchi Y. Diffusion-mediated persistence in two-species competition Lotka-Volterra model. Mathematical Biosciences. 1989; 95: 65.

[7] Zhang XA, Chen L. The linear and nonlinear diffusion of the competitive Lotka-Volterra model ţ. Nonlinear Analysis Theory Methods \& Applications. 2007; 66: 2767-2776.

[8] Wei F, Wang K. Asymptotically periodic solution of N-species cooperation system with time delay 2 . Nonlinear Analysis Real World Applications. 2006; 7: 591-596.

[9] Dareiotis KA, Gyöngy I. A Comparison Principle for Stochastic IntegroDifferential Equations. Potential Analysis. 2014; 41: 1203-1222. 